\title{
Niveles de contaminación en tres sectores de Villavicencio, a través del índice de geo-acumulación(I-geo)
}

\section{Contamination levels in three sectors of Villavicencio using the geoaccumulation (Igeo) index}

\section{Niveis de contaminação em três setores de Villavicencio, a través do índice de Geo- Acumulação (I-Geo)}

\section{Juan M. Trujillo-González ${ }^{1 *}$; Marco A. Torres-Mora ${ }^{2 *}$}

\footnotetext{
${ }^{1}$ Ingeniero Agrónomo, MSc; ${ }^{2}$ Biólogo, PhD

* Grupo de Investigación en Gestión Ambiental Sostenible -GIGAS-. Facultad de Ciencias Básicas e Ingenierías. Instituto de Ciencias Ambientales de la Orinoquia Colombiana. Universidad de los Llanos.

Email: jmtrujillo18@gmail.com
}

Recibido: febrero 27 de 2015

Aceptado: abril 27 de 2015

\section{Resumen}

La contaminación urbana producto de la dinámica de las ciudades ha generado interés en los últimos años. El presente estudio tiene como objetivo identificar la contaminación de los metales pesados ( $\mathrm{Pb}, \mathrm{Cd}, \mathrm{Cu}, \mathrm{Cr}, \mathrm{Ni}, \mathrm{Zn}$ ) en tres sectores de la ciudad de Villavicencio, a través de la aplicación del índice de geo-acumulación (I-geo). La determinación de metales pesados se realizó mediante espectrofotometría de absorción atómica. Como resultados se encontró que las mayores concentraciones de estos metales, especialmente el plomo, estuvieron en el sector Porvenir, zona asociada a la actividad de mecánica automotriz "rudimentaria". Los sedimentos viales son un factor que aumentan con el crecimiento y la dinámica de las ciudades y pueden ser responsables de numerosos impactos ambientales.

Palabras Claves: Metales pesados, contaminación urbana, Plomo Sedimento vial

\begin{abstract}
Urban contamination produced by city dynamics has aroused interest during the last few years. This study involved using the (Igeo) geoaccumulation index for identifying the contamination produced by heavy metals (i.e. $\mathrm{Pb}, \mathrm{Cd}, \mathrm{Cu}, \mathrm{Cr}, \mathrm{Ni}, \mathrm{Zn}$ ) in three sectors of the city of Villavicencio; heavy metal concentration was determined by atomic absorption spectroscopy (AAS). It was found that the greatest concentrations of these metals, especially lead, occurred in the Porvenir sector of the city, this being an area associated with rudimentary car repair and wrecker activity. Road deposited sediment (RDS) is a factor having an impact on cities' increased growth and dynamics and could be responsible for many environmental issues.
\end{abstract}

Key words: heavy metal, urban pollution, lead concentration in road deposited sediment (RDS). 


\section{Resumo}

A poluição urbana produto da dinâmica das cidades tem gerado interesse nos últimos anos. Este estudo tem como objetivo identificar a contaminação de metais pesados ( $\mathrm{Pb}, \mathrm{Cd}, \mathrm{Cu}, \mathrm{Cr}, \mathrm{Ni}, \mathrm{Zn})$ em três setores da cidade de Villavicencio, através da aplicação do índice de geo-acumulação (I-geo ). A determinação de metais pesados foi realizada utilizando a espectrometria de absorção atómica. Como resultado, verificou-se que as maiores concentrações destes metais, especialmente chumbo, estavam na atividade do setor Porvenir associada à área " mecânica rudimentar de automóveis. Os sedimentos nas ruas são um factor que aumenta com o crescimento e a dinâmica das cidades e podem ser responsáveis por muitos impactos ambientais.

Palavras-chave: metais pesados, poluição urbana, sedimentos, chumbo

\section{Introducción}

El crecimiento acelerado de las grandes ciudades ha cesado en los últimos cincuenta años. Sin embargo, en la última década las pequeñas y medianas ciudades han crecido y afrontan nuevos retos como los efectos relacionados con el aumento poblacional, la expansión urbana sin planificación, la carencia de red de servicios y en general en la dinámica de las relaciones económicas y demográficas que en ellas se establecen (Stren, 1995; Borja, 2003). En cuanto a la expansión de barrios, de áreas comerciales e industriales y la construcción de infraestructura vial, puede traer consigo potenciales impactos negativos como deficientes servicios urbanos, sobrecarga del tránsito vehicular, barreras a la circulación urbana y alteración de la escorrentía de aguas superficiales, entre otros.

La densidad del tránsito vehicular sumada a factores como las emisiones provenientes de la industria y en general de la actividad económica de las ciudades, ha convertido a las vías en uno de los principales receptores de contaminantes que constituyen potenciales problemas de salud pública para los usuarios de las vías, de los habitantes aledaños, y a la dinámica ecológica de los sistemas naturales (Brown y Peake, 2006; Bae et al., 2006; Aelion et al., 2009). Los sedimentos acumulados en zonas urbanas tienen una diversidad de materiales que van desde compuestos minerales y materiales biogénicos a materiales orgánicos e inorgánicos de origen antropogénico que se pueden depositar sobre las superficies impermeables de las ciudades, como son las vías y techos (Shi et al., 2010).

Davis y Birch (2010), argumentan que en las cuencas urbanizadas, las vías pueden constituir un $22 \%$ del área y éstas contribuyen con el $26 \%$ de la escorrentía. Sartor y Boyd (1974), determinaron que la carga de contaminantes de las aguas de escorrentía urbana es significativamente mayor en comparación con la escorrentía rural. Esto determina, que gran parte de la contaminación de las fuentes hídricas urbanas y de los suelos aledaños estén relacionadas con los aportes viales (Vaze y Chiew, 2002). Rissler et al., (2012), encon- traron que los principales contaminantes asociados a las cuencas urbanizadas son el Cobre $(\mathrm{Cu})$, Plomo $(\mathrm{Pb})$ y Zinc (Zn); junto a estos también pueden estar el Níquel $(\mathrm{Ni})$, Cadmio $(\mathrm{Cd})$ y Cromo $(\mathrm{Cr})$, provenientes de las actividades industriales (García y Poleto, 2014).

El sedimento urbano acumulado en las vías es un sumidero de contaminantes en las ciudades, como los metales pesados, donde el contacto y la ingestión de partículas provenientes de éstos, puede traer graves problemas para la salud humana (Zhenget al., 2010; Acosta et al., 2014). Con base en lo anterior, el monitoreo de vías que presentan alto flujo vehicular y que además están en zonas industriales, debe ser una tarea prioritaria en los programas de salud pública y de gestión del riesgo (Nazzal et al., 2012). Este tipo de estudios se han realizado especialmente en países de oriente como China (Yekeen y Onifade, 2012), y también en otros como Egipto (Khairy et al., 2011), Nigeria (Abdel-Latif y Saleh, 2012), Irán (Saeedi et al., 2012), Ghana (Atiemo et al., 2011), España (Acosta et al., 2014) y Brasil (García y Poleto, 2014). Iones metálicos como el $\mathrm{Pb}, \mathrm{Cu}, \mathrm{Cd}$ y $\mathrm{Zn}$ se relacionan principalmente con el uso de combustibles tipo gasolina, desgaste de neumáticos y de pastillas de frenos, de aceites, de lubricantes y grasas (Christoforidis y Stamatis, 2009); mientras que $\mathrm{Cr}$ y el $\mathrm{Ni}$, provienen del desgaste de partes metálicas y del cromado de accesorios (Al-Shayep y Seaward, 2001). En Colombia los estudios relacionados con metales pesados han sido enfocados hacia los sedimentos acuáticos continentales y marinos, mientras que para los sedimentos viales aún son escasos y se destacan los realizados por Zafra, en el municipio de Soacha (Cundinamarca), donde analiza los procesos de contaminación con la influencia del tráfico, la asociación de los metales en polvo atmosférico con los presentes en los sedimentos viales y la contaminación por procesos de escorrentía urbana (Zafra et al., 2013a; Zafra et al., 2013b; Zafra et al., 2013c; Zafra et al., 2009).

Para la evaluación del grado de contaminación por metales pesados presentes en sedimentos, Müller (1969), propone el índice de geo-acumulación (I-geo), donde a través de la comparación entre los valores actuales y 
los valores de referencia, es decir los contenidos que naturalmente tendría la región, se evalúa la presencia de éstos. Autores como Khairy et al., (2011), García y Poleto (2014), entre otros, han aplicado este índice como herramienta para evaluar la contaminación urbana, como resultado de la presencia de metales pesados en los sedimentos viales.
La ciudad de Villavicencio por su ubicación geográfica (punto de conexión entre los llanos orientales y el centro del país) cumple un rol fundamental en el desarrollo económico del país, con un crecimiento urbanístico, un aumento en el parque automotor, $y$ una mayor oferta y demanda de bienes y servicios (Alcaldía de Villavicencio, 2013); que por ende han traído consigo
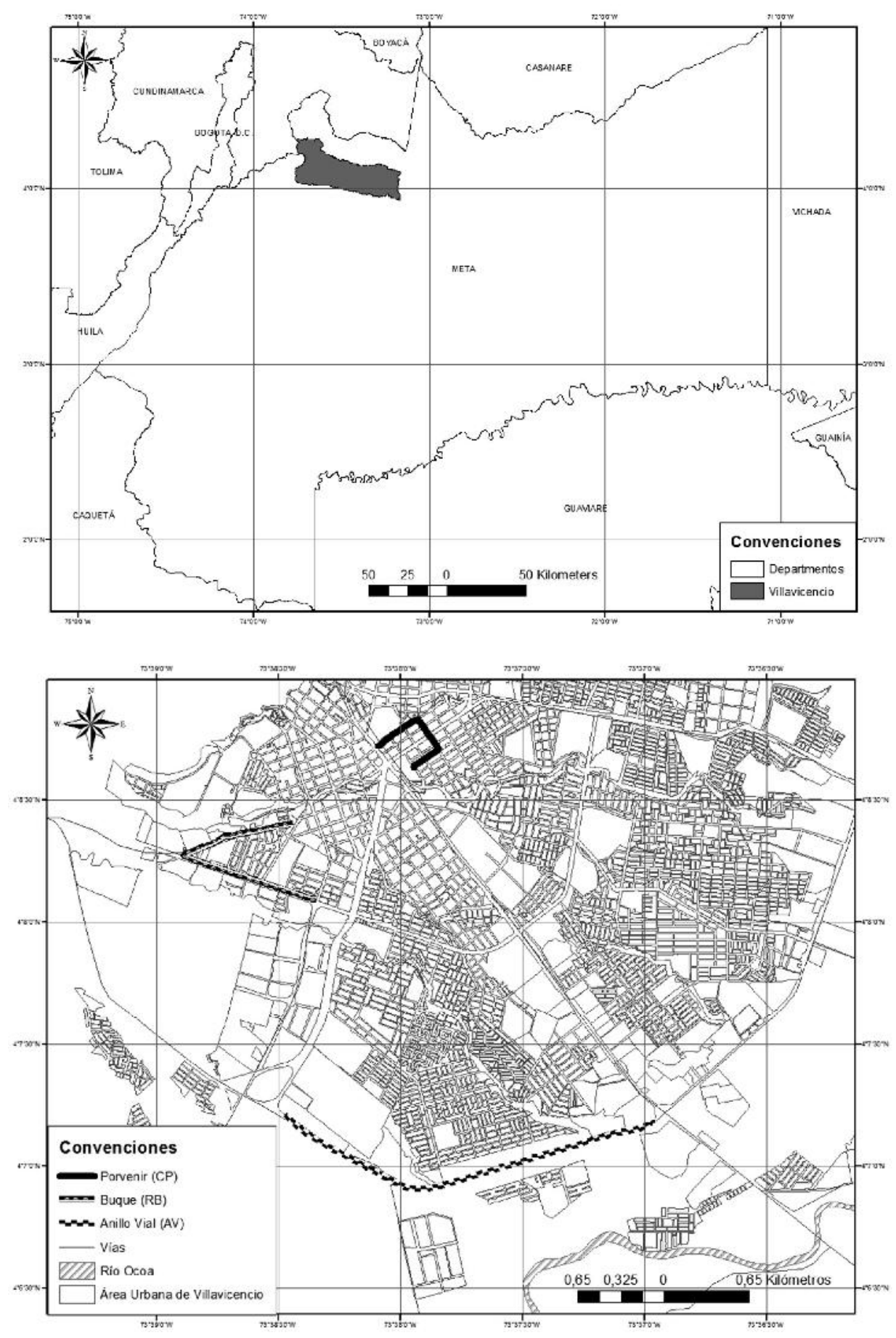

Figura 1. Sectores de muestreo en el área urbana de la ciudad de Villavicencio. 
problemáticas propias como la contaminación urbana. En este contexto, el presente trabajo tiene como objetivo identificar la contaminación de los metales pesados $(\mathrm{Pb}, \mathrm{Cd}, \mathrm{Cu}, \mathrm{Cr}, \mathrm{Ni}, \mathrm{Zn})$ en tres sectores de la ciudad de Villavicencio, a través de la aplicación del índice de geo-acumulación (I-geo).

\section{Materiales y métodos}

\section{Área de estudio}

El área de estudio se localizada en el área urbana de la ciudad de Villavicencio en el departamento del Meta (Figura 1), que presenta condiciones de temperatura promedio anual de $25,6{ }^{\circ} \mathrm{C}$, una precipitación media anual registrada de $3.700 \mathrm{~mm}$, una altura sobre el nivel del mar de 467 metros y con una población estimada para el año 2014 en 473.718 habitantes (DANE, 2010). Dentro del área de estudio, se presentan los sectores donde se desarrollará el trabajo, una avenida principal (Anillo vial), un sector residencial (Buque) un sector comercial en el centro de la ciudad (Porvenir); los tres sectores seleccionados hacen parte de la cuenca del río Ocoa. El sector del Anillo vial (AV) se caracteriza porque en él circula todo el tráfico pesado de transporte de productos alimenticios e hidrocarburos, así como el transporte intermunicipal desde y hacia Bogotá D.C., además del transporte particular. También se desarrollan actividades económicas tales como, venta de automotores y asistencia mecánica, estaciones de servicios, oficinas y bodegas de bebidas gaseosas y de agroquímicos, al igual que áreas urbanizadas. El sector Buque (RB) se ubican áreas residenciales de estratos altos, restaurantes y centros educativos; mientras que en el Porvenir (CP) se encuentra un área comercial donde la principal actividad está relacionada con la venta de autopartes y servicios de mecánica automotriz sin tecnificación.

\section{Muestreo de sedimentos viarios}

Se recogieron un total de 15 muestras de forma compuesta del sedimento acumulado en las calles de los sectores estudiados, cada muestreo se realizó con un intervalo de 7 días, para un total de cinco muestras por sector. Para la recolección de las sub-muestras se utilizó un marco de $0,25 \mathrm{~m}^{2}$, brochas de 3 pulgadas y recogedores plástico de mano; para cada sitio se utilizaron distintos utensilios. El material recolectado se homogenizó utilizando un tamiz de 2,0 mm, con el objetivo de retirar impurezas de mayor tamaño como piedras, puntillas o material orgánico, y se tomó 200 gramos por muestra de sedimento vial que fue empacado en bolsas selladas. Las campañas de muestreo se realizaron entre los meses de marzo - abril de 2014

\section{Análisis de laboratorio}

La detección de las concentraciones de los metales pesados totales ( $\mathrm{Cu}, \mathrm{Pb}, \mathrm{Ni}, \mathrm{Zn}, \mathrm{Cr})$, se realizó de acuerdo con el método de digestión ácido nítrico, ácido clorhídrico y peróxido de Hidrógeno, Espectrofotometría de Absorción Atómica con llama Aire - Acetileno, EPA 3050 B, SM 3111 B. Los límites de detección fueron: $\mathrm{Pb} \mathrm{1,5mg/L;} \mathrm{Ni} \mathrm{0,12} \mathrm{mg/L;} \mathrm{Zn} \mathrm{0,010} \mathrm{mg/L;} \mathrm{Cu} \mathrm{0,038}$ $\mathrm{mg} / \mathrm{L}$; $\mathrm{Cr}$ 0,076 mg/L. Todos los análisis se realizaron en el laboratorio químico de consultas industriales de la Universidad Industrial de Santander, acreditado por el IDEAM según resolución 1659 de 2011.

\section{Índice de Geo-acumulación (I-geo)}

El índice de geo-acumulación (I-geo) propuesto por Müller (1969), es ampliamente utilizado para evaluar la contaminación a través de la relación de la concentración presente frente a los valores de referencia.

$$
I-g e o=\log _{2}\left[\left[\left(\frac{C S}{1.5 \times B n}\right)\right]\right]
$$

El factor 1.5 se aplica para disminuir las variaciones en los valores de fondo ocasionado por el entorno, $\mathrm{Cs}$ se refiere a los valores calculados y $\mathrm{Bn}$ a los valores de referencia, en este caso se utilizaron los propuesto por Fadigas et al., (2006). En la Tabla 1 se presentan la categorías de interpretación.

\section{Resultados y discusión}

En la tabla 2, se presentan los resultados obtenidos en cuanto a la concentración de metales pesados en sedimento vial de los tres sectores estudiados y se comparan a su vez con los valores de referencia asumidos, al igual que el índice de geo-acumulación encontrado para cada uno de los sectores.

\section{Concentración de metales pesados}

En la tabla 2, se presentan los resultados de los metales pesados en los sedimentos viales, estos varían ampliamente entre los sectores de muestreo y entre las muestras, debido a la heterogeneidad de éstas y a las múltiples variables que no pueden ser controladas entre cada periodo de muestreo, lo cual puede explicarse debido al alto grado variabilidad de los metales independientemente de la actividades económicas propias de cada sector. En todos los sectores se destaca la presencia de $\mathrm{Pb}, \mathrm{Cu}$ y $\mathrm{Zn}$, lo que corrobora lo mencionado por García y Poleto (2014), en estudios similares. Los resultados más sobresalientes son los del $\mathrm{Pb}$, donde se detectaron para el sector CP $1289,4 \mathrm{mg} /$ 
Tabla 1. Categorías de evaluación del Índice de Geoacumulación

\begin{tabular}{|l|l|}
\hline \multicolumn{1}{|c|}{ Valor } & \multicolumn{1}{c|}{ Categoría } \\
\hline I-geo $\leq 0$ & No contaminado \\
\hline $0<$ I-geo $<1$ & $\begin{array}{l}\text { No contaminado a } \\
\text { moderadamente contaminado }\end{array}$ \\
\hline $1<$ I-geo $<2$ & Moderadamente contaminado \\
\hline $2<$ I-geo $<3$ & $\begin{array}{l}\text { Moderadamente a altamente } \\
\text { contaminado }\end{array}$ \\
\hline $3<$ I-geo $<4$ & Altamente contaminado \\
\hline $4<$ I-geo $<5$ & $\begin{array}{l}\text { Altamente a extremadamente } \\
\text { contaminada }\end{array}$ \\
\hline I-geo $\geq 5$ & Extremadamente contaminado \\
\hline
\end{tabular}

$\mathrm{Kg}$ en promedio, superando ampliamente el valor tomado como referencia de $17 \mathrm{mg} / \mathrm{Kg}$ (Fadigas et al., 2006), del mismo modo el sector sobrepasa los valores máximos permisibles planteados para sectores comerciales de México $400 \mathrm{mg} / \mathrm{Kg}$ (NOM-147-SEMARNAT/ SSA1, 2004) y para Argentina de $500 \mathrm{mg} / \mathrm{Kg}$ (Decreto 831 1993). En el caso del sector $A V$ el promedio de $\mathrm{Pb}$ fue de $87,5 \mathrm{mg} / \mathrm{Kg}$ y para el sector RB de $26 \mathrm{mg} / \mathrm{Kg}$; aunque superan los valores de referencia se mantienen por debajo de los valores permisibles en sectores residenciales y comerciales propuestos en México y Argentina.

Los valores encontrados de $\mathrm{Pb}$ en el sector $\mathrm{CP}$ están por encima de lo que Zafra et al., (2013b), reporta para el corredor vial Soacha - Bogotá, que están entre 81 y $202 \mathrm{mg} / \mathrm{Kg}$, y de los reportados por García y Poleto (2014), para Porto Alegre de 655 mg/Kg, ambas ciudades con una población superior a la de Villavicencio, que alcanza alrededor de 500.000 habitantes.

\section{Índice de geo-acumulación}

La aplicación de índice de geo-acumulación que permite identificar el grado de contaminación del sedimento vial por la acumulación de metales, para el caso de $\mathrm{Vi}$ llavicencio se encontró que las categorías de contaminación se relacionaron con en el sector CP y para los metales $\mathrm{Pb}, \mathrm{Cu}$ y $\mathrm{Zn}$; mientras que en los demás sectores predominaron las categorías de no contaminado, la razón de esto se asocia a la actividad comercial del sector $\mathrm{CP}$, donde predomina la prestación de servicios de mecánica automotriz con tecnología "rudimentaria" y escasos protocolos para la gestión adecuada de

Tabla 2. Concentración de metales pesados en sedimentos comparados con valores de referencia e índice de geo-acumulación de tres sectores de la ciudad de Villavicencio Colombia.

\begin{tabular}{|c|c|c|c|c|c|c|c|c|c|c|c|}
\hline \multirow{2}{*}{\multicolumn{2}{|c|}{ Parámetro }} & \multicolumn{5}{|c|}{ Concentraciones en $\mathrm{mg} / \mathrm{Kg}$} & \multicolumn{5}{|c|}{ I-geo } \\
\hline & & \multirow{2}{*}{$\frac{\mathbf{P b}}{87,5}$} & \multirow{2}{*}{$\frac{\mathbf{N i}}{5,3}$} & \multirow{2}{*}{$\frac{\mathbf{Z n}}{133,3}$} & \multirow{2}{*}{$\frac{\mathbf{C u}}{126,3}$} & \multirow{2}{*}{$\frac{\mathbf{C r}}{9,4}$} & \multirow{2}{*}{$\frac{\mathbf{P b}}{0,8}$} & \multirow{2}{*}{$\frac{\mathbf{N i}}{-2,1}$} & \multirow{2}{*}{$\frac{\mathbf{Z n}}{0,5}$} & \multirow{2}{*}{$\frac{\mathbf{C u}}{0,9}$} & \multirow{2}{*}{$\frac{\mathbf{C r}}{-2,7}$} \\
\hline Avenida & Media & & & & & & & & & & \\
\hline principal & Mínimo & 26,4 & 3,0 & 85,6 & 24,9 & 6,1 & 0,0 & $-2,7$ & $-0,1$ & $-1,1$ & $-3,3$ \\
\hline $\begin{array}{l}\text { (Anillo } \\
\text { Vial_ AV) }\end{array}$ & Máximo & 326,2 & 10,4 & 189,8 & 248,1 & 14,0 & 3,7 & $-0,9$ & 1,1 & 2,2 & $-2,1$ \\
\hline & SD & 133,5 & 3,2 & 53,5 & 91,5 & 3,0 & 1,6 & 0,8 & 0,6 & 1,3 & 0,4 \\
\hline \multirow{4}{*}{$\begin{array}{l}\text { Residencial } \\
\text { (Buque- RB) }\end{array}$} & Media & 26,0 & 7,2 & 108,3 & 23,7 & 7,3 & 0,0 & $-1,5$ & 0,3 & $-1,3$ & $-3,1$ \\
\hline & Mínimo & 17,5 & 6,5 & 104,4 & 13,6 & 4,7 & $-0,5$ & $-1,7$ & 0,2 & $-2,0$ & $-3,7$ \\
\hline & Máximo & 31,7 & 8,9 & 113,8 & 40,0 & 10,0 & 0,3 & $-1,2$ & 0,3 & $-0,4$ & $-2,6$ \\
\hline & SD & 4,7 & 0,9 & 4,1 & 9,6 & 1,9 & 0,3 & 0,2 & 0,1 & 0,6 & 0,4 \\
\hline \multirow{4}{*}{$\begin{array}{l}\text { Comercial } \\
\text { (PorvenirCP) }\end{array}$} & Media & 1289,4 & 54,3 & 387,6 & 490,2 & 60,2 & 4,9 & 1,2 & 2,0 & 2,6 & $-0,3$ \\
\hline & Mínimo & 259,2 & 20,7 & 222,5 & 36,1 & 27,1 & 3,3 & 0,1 & 1,3 & $-0,5$ & $-1,5$ \\
\hline & Máximo & 4079,8 & 123,3 & 511,8 & 903,0 & 115,3 & 7,3 & 2,6 & 2,5 & 4,1 & 0,9 \\
\hline & $\mathrm{SD}$ & 1598,8 & 41,3 & 129,0 & 345,9 & 35,7 & 1,6 & 1,0 & 0,6 & 1,9 & 1,0 \\
\hline Referencia* & & 17,0 & 13,2 & 59,9 & 35,1 & 40,2 & & & & & \\
\hline
\end{tabular}

* Valores de referencia o backgroud de metales pesados en suelos propuestos por Fadigas et al., (2006) 

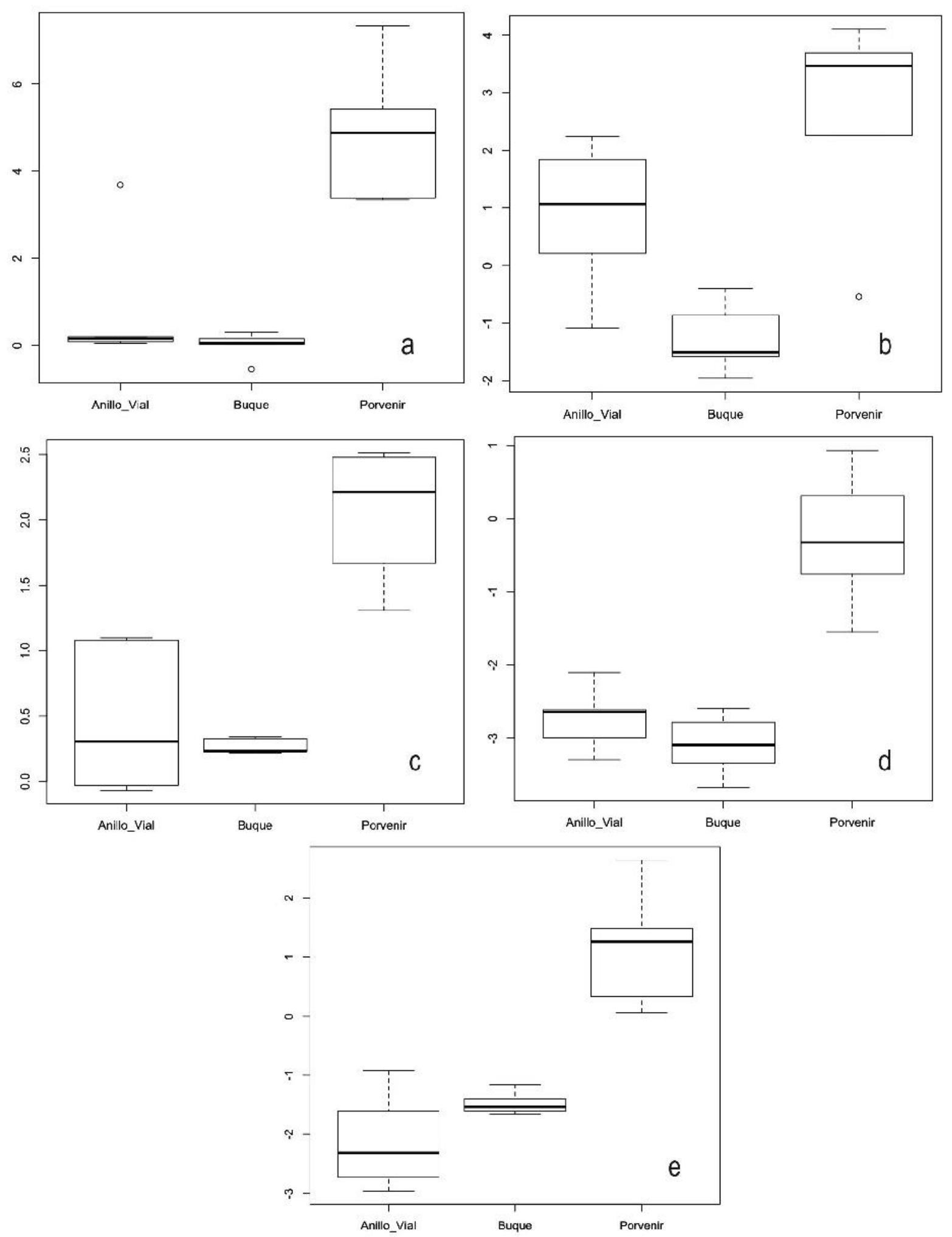

Figura 2. Diagrama de cajas del índice de geo-acumulación (I-geo) en los sectores de estudio. $a=$ Plomo. $b=$ Cobre. $c=Z$ Zinc. $d=$ Cromo. e= Níquel 
residuos como aceites, grasas, pinturas, combustibles y limaduras metálicas, entre otros.

En la figura 2, se presentan diagramas de cajas con los índices de geo-acumulación para los metales objeto de estudio. Los valores de $\mathrm{Pb}$ en los sectores $\mathrm{CP}$ con valor I-geo de 4,9 superan lo encontrado por García y Poleto (2014) en la ciudad de Porto Alegre-Brasil, donde el I-geo para Pb fue de 1,75, a pesar que esta ciudad tiene una población superior al millón de habitantes. $\mathrm{De}$ igual forma, este valor de l-geo para $\mathrm{Pb}$ en el sector CP también supera lo reportado para China, 2,32Igeo (Shiet al., 2010) y 1,81lgeo (Wei y Yang, 2010). En el caso del Cu el I-geo fue de 4,1 en el sector CP, que también se supera el valor encontrado en China de 1,48 (Shi et al., 2010), y el Zn, aunque el mayor valor de l-geo para el sector CP en Villavicencio fue de 1,3, está por debajo de lo reportando para Porto Alegre 3,04 (García y Poleto, 2014) y en China 2,44 (Shi et al., 2010), De acuerdo con el índice de geo-acumulación el sector CP están en las categorías moderadamente a altamente contaminado por la presencia de $\mathrm{Pb}, \mathrm{Cu}$ y Zn. Para los sectores AV y RB la concentración que presentan de metales pesados los llevan a las categoría de No contaminado a Moderadamente. La presencia de estos metales en el sedimento vial genera alto riesgos para la salud pública, debido a que fácilmente la población puede intoxicarse al adquirirlo a través de inhalación, cuando el polvo es levantado por efecto del viento o del tráfico vehicular, acarreando efectos patológicos como la anemia hemolítica, las neuropatías y encefalopatías (Charris et al., 2011), y para el caso de los sistemas naturales especialmente las fuentes hídricas que pueden verse afectada por efecto de la escorrentía urbana, debido a que ésta puede arrastrar los metales y otros contaminantes presentes en las vías, especialmente en ciudades con altas precipitaciones como Villavicencio.

En respuesta a la problemática de residuos que se generan en las vías de los centros urbanos y ciudades, la Organización Mundial de la Salud (Fernández, 2002), plantea que "El servicio de limpieza urbana es un tema de creciente preocupación para los gobiernos locales y la adecuada disposición final de los residuos sólidos es un imperativo para las ciudades que deseen un hábitat saludable". En la ciudad de Villavicencio, se implementa un sistema de limpieza y barrido de vías que se realiza de forma manual y mecánica, con frecuencias de dos veces por semana en el sector residencial y diariamente en los sectores comerciales y avenidas principales. Aunque actividad es realizada conforme a lo que plantea el Decreto 2981 de 2013, donde se establecen los lineamientos para la gestión de sedimentos viales o arenillas. Sin embargo, en la normatividad colombiana se desconoce el potencial contaminante que tienen los sedimentos viales o arenillas y no se plantean prácticas para evitar la posible afectación ambiental y de salud pública.

En este sentido, el modelo actual de limpieza y barrido de vías, no tiene factores diferenciales que permitan gestionar estos residuos dependiente del sector donde es generado, lo que puede conducir a potenciales de riesgo debido a la transferencia de contaminantes de un sitio a otro. Caso puntal el sector CP en la ciudad de Villavicencio, donde a través del índice geo-acumulación se identificó con categorías de moderadamente a altamente contaminado por las concentraciones que presenta de $\mathrm{Pb}, \mathrm{Cu}$ y Zn. En este sentido, es necesario que se plante un sistema diferencial de gestión de residuos sólidos, puntalmente de los sedimentos viales, donde se garantice que los elementos tales como: escobas, palas, rastrillo, escobillas, sean específicos para sectores con dinámicas socioeconómicas similares. Así mismo, es necesario que este sistema considere la disposición final del sedimento, debido que al mezclarse con los residuos sólidos convencionales podría aumentar la problemática de los lixiviados provenientes del material orgánico recolectado.

\section{Conclusiones}

Los sedimentos viales son un factor que aumentan con el crecimiento y la dinámica de las ciudades y pueden ser responsables de numerosos impactos ambientales. En la ciudad de Villavicencio se encontró que las mayores concentraciones de estos metales, especialmente el plomo, estuvieron en el sector Porvenir (CP), zona asociada a la actividad de mecánica automotriz "rudimentaria".

A través de la aplicación del índice de geo-acumulación se identificó también que el sector del Porvenir $(\mathrm{CP})$, se encuentra en las categorías de moderadamente a altamente contaminado y que los metales pesados que llevan a esta categorización son el $\mathrm{Pb}, \mathrm{Cu}$ y Zn.

Por otro lado, el actual modelo de limpieza de las vías en la ciudad de Villavicencio puede convertirse en un factor de riesgo de contaminación debido a que existen sectores, como el Porvenir, que por su dinámica económica presenta una carga de contaminantes mayor a la de otros, específicamente concentraciones elevadas de metales pesados, y que al no contar con un sistema diferencial de limpieza se podría estar transportando contaminantes de un sector a otro. Además su disposición indiscriminada en el relleno sanitario podría generar problemas ambientales en otros sis- 
temas por el arrastre de los metales en los lixiviados producidos.

\section{Agradecimientos}

Los autores agradecen el apoyo financiero de Ecopetrol S.A., mediante el convenio con la Universidad de los Llanos № 5211592, y al personal del Instituto de Ciencias Ambientales de la Orinoquia Colombiana de la Universidad de los Llanos.

\section{Referencias}

Abdel-Latif NM, Saleh IA. Heavy Metals Contamination in Roadside Dust along Major Roads and Correlation with Urbanization Activities in Cairo, Egypt. Journal of American Science. 2012;8(6):379-389.

Aelion CM, Davisa HT, Mc Dermottb S, Lawson AB. Soil metal concentrations and toxicity: associations with distances to industrial facilities and implications for human health. Sci. Total Environ.2009; 407, 216-223.

Acosta JA, Faz A, Kalbitz K, Jansen y Martínez-Martínez S. Partitioning of heavy metals over different chemical fraction in street dust of Murcia (Spain) as a basis for risk assessment. Journal of Geochemical Exploration. 2014; 144(Part B): 298-305.

Alcaldía de Villavicencio. 2013 Formulación del plan de movilidad para el municipio de Villavicencio. Informe Diagnostico № 2 .

Al-Shayep SM, Seaward MRD. Heavy metal content of roadside soils along ring road in Riyadh (Saudi Arabia). Asian Journal of Chemistry. 2013; 13: 407-423

Atiemo MS, OfosuGF, Kuranchie-Mensah H, Tutu AO, Palm ND, Blankson SA. Contamination assessment of heavy metals in road dust from selected roads in Accra, Ghana. Research Journal of Environmental and Earth Sciences. 2011;3(5): 473480 .

Bae S, Inyang HI, De Brito TC, Mbamalu GE. Soil desiccation rate integration into empirical dust emission models for polymer suppressant evaluation. J. Hazard. Mater. 2006; 132: 111-117.

Borja J. 2003. Ciudad y planificación. Balbo, M., Jordán, R. Y Simioni, D. (compiladores). La ciudad inclusiva. Santiago. Cuadernos de la CEPAL. 88:81-104.

Brown JN, Peake BM. Sources of heavy metals and polycyclic aromatic hydrocarbons in urban stormwater runoff. Sci. Total Environ. 2006; 359: 145-155.

Charris V, Guerrero A, Barrera C. Intoxicación por plomo secundaria a alojamiento de esquirlas en el cuerpo. Acta Médica Colombiana. 2011;36(4): 200-203.

Christoforidis A, Stamatis N.Heavy metal contamination in street dust and roadside soil along the major national road in Kavala's region, Greece. Geoderma. 2009;151(3): 257-263.

COLOMBIA. 2013. Decreto 2981/2013, de 20 de Diciembre, por el cual se reglamenta la prestación del servicio público de aseo.
Publicación en el Diario Oficial 49010 de diciembre 20 de 2013.

DANE. 2010. Proyecciones demográficas por municipio basadas en el censo del año 2005. Recuperado de www.dane.gov.co/files/ investigaciones/poblacion/proyepobla06_20/ProyeccionMunicipios2005_202 0.xls

Davis B, Birch G. Comparison of heavy metal loads in stormwater runoff from major and minor urban roads using pollutant yield rating curves. Environmental Pollution. 2010; 158(8): 25412545.

Decreto 831/1993, Decreto Reglamentario de la Ley 24.051 sobre régimen de desechos peligrosos, Niveles guía de calidad suelos (ug/g peso seco). Argentina 1993.

Fadigas FD, Amaral-Sobrinho NMB, Mazur N, Anjos LHC, Freixo AA. Proposição de valores de referência para a concentração natural de metais pesados em solos brasileiros. R Bras Eng Agríc Ambiental. 2006;10(3):699-705.

Fernández D. 2002. Guía para la Regulación de los Servicios de Limpieza Urbana. Centro Panamericano de Ingeniería Sanitaria y Ciencias del Ambiente. Organización Panamericana de la Salud. Lima.

García-Martínez LL, Poleto C. Assessment of diffuse pollution associated with metals in urban sediments using the geoaccumulation index (Igeo). Journal of Soils and Sediments. 2014;14(7):1251-1257.

Khairy MA, Barakat AO, Mostafa AR, Wade TL. Multielement determination by flame atomic absorption of road dust samples in Delta Region, Egypt. Microchemical Journal. 2011; 97(2): 234-242.

Müller G. Index of geoaccumulation in sediments of the Rhine River. Geo journal. 1969; 2:108-118.

Nazzal Y, Ghrefat H, Rosen MA. Application of multivariate geostatistics in the investigation of heavy metal contamination of roadsidedusts from selected high ways of the Greater Toronto Area, Canada. Environ mentalearth sciences. 2014; 71(3): 1409-1419.

NOM-147-SEMARNA T/SSA1-2004. Diario Oficial. 2 de Marzo de 2007. Segunda Edición.

Rissler J, Swietlicki E, Bengtsson A, Boman C, Pagels J, Sandstrom T, Blomberg A, Londahl J. Experimental determination of deposition of diesel exhaust particles in the human respiratory tract. Journal of Aerosol Science. 2012; 48:18-33.

Saeedi M, Li L, Salmanzadeh M. Heavy metals and polycyclic aromatic hydrocarbons: pollution and ecological risk assessment in street dust of Tehran. Journal of hazardous materials. 2012; 227: 9-17.

Sartor JD, Boyd GB, Agardy FJ. Water pollution aspects of street surface contaminants. Journal (Water Pollution Control Federation), 1974;46(3): 458-467.

Shi G, Chen Z, Bi C, Li Y, Teng J, Wang L, Xu S. Comprehensive assessment of toxic metals in urban and suburban street deposited sediments (SDSs) in the biggest metropolitan area of China. Environmental pollution. 2012; 158(3): 694-703. 
Vaze J, ChiewFH. Experimental study of pollutant accumulation on an urban road surface. Urban Water. 2002; 4(4): 379-389.

Wei B, Yang L. A review of heavy metal contaminations in urban soils, urban road dusts and agricultural soils from China. Microchemical Journal. 2010; 94(2): 99-107.

Yekeen TA, Onifade TO. Evaluation of some Heavy Metals in Soils along a Major Road in Ogbomoso, South West Nigeria. Journal of Environment \& Earth Science. 2012; 2(8): 71-79.

Zafra-Mejía CA, Luengas-Pinzón EC, Temprano-González J. Influencia del tráfico en la acumulación de metales pesados sobre vías urbanas: Torrelavega (España)-Soacha (Colombia). Revista Facultad de Ingeniería. 2013a; 67:146-160.

Zafra-Mejía CA, Peña-Valbuena NÁ, Álvarez-Prieto S. Contaminación por metales pesados en los sedimentos acumulados sobre el corredor vial Bogotá-Soacha. Tecnura. 2013b; 17(37):99108.

Zafra-Mejía CA, Rodríguez-Chitiva LG, Torres-Cabrera YA. Metales pesados asociados con las partículas atmosféricas y sedimentadas de superficies viales: Soacha (Colombia). Revista Científica. 2013c; 17: 113-124.

Zafra-Mejía CA, Temprano-González J, Tejero-Monzón I. Evaluación de la contaminación por escorrentía urbana: sedimentos depositados sobre la superficie de una vía. Ingeniería e Investigación. 2009; 29(1):101-108.

Zheng N, Liu J, Wang Q, Liang Z. Health risk assessment of heavymetal exposure to street dust in the zinc smelting district, Northeast of China. Sci. Total Environ. 2010; 408: 726-733. 